\title{
Pilot study to differentiate lipoma from atypical lipomatous tumour/well differentiated liposarcoma using MR radiomics-based texture analysis.
}

\begin{abstract}
Aims This pilot study aims to determine if tumour heterogeneity assessed using magnetic resonance imaging (MRI) radiomics-based texture analysis (TA) can differentiate between lipoma and atypical lipomatous tumour (ALT)/well differentiated liposarcoma (WDL).
\end{abstract}

Materials and Methods 30 consecutive ALT/WDLs and 30 lipomas were included in the study, cases diagnosed both histologically and with murine double minute 2 (MDM2) gene amplification by fluoresence in situ hybridisation (FISH) in excision specimens. Multiple patient, MRI and MRTA factors were assessed. Heterogeneity was evaluated using a filtration-histogram technique based textural analysis on single axial proton density (PD) and coronal T1-W images of the most homogenously fatty component of the lesion.

Results $33 \%$ of the diagnoses of ALT/WDL vs lipoma were confirmed using FISH MDM2 analysis.. ALT/WDLs were statistically different from lipomas in location (site in the body and depth from skin surface) and fat content, with $\mathrm{p}$ values of $0.021,0.001$, and 0.021 respectively. 9 of $36(25 \%)$ texture parameters had significant differences between ALT/WDLs and lipomas on axial PD MRTA, with the most significant results at medium and coarse texture scales particularly mean intensity $(\mathrm{p}=0.003)$ at $\mathrm{SSF}=6$, and kurtosis $(\mathrm{p}=0.012)$ at $\mathrm{SSF}=5$. A cut-off value of $<304$ for coarse filtered texture on axial PD MRI identified ALT from lipoma with a sensitivity and specificity of $70 \%$ (AUC=0.73, $\mathrm{p}=0.003$ ). 
Conclusions Texture heterogeneity quantified at fine, medium, coarse texture scales are significant differentiators of lipoma and ALT/WDL with the difference particularly marked in medium and coarse texture scales for two MR TA parameters: mean and kurtosis. 


\section{Introduction}

Fat containing tumours are commonly encountered in clinical practice (1). Lipomas are the most common soft tissue tumours and liposarcomas the most common soft tissue sarcoma (2). Lipomas are benign and do not require surgical excision or formal surveillance. The World Health Organization (WHO) committee for Classification of Soft Tissue Tumours in 2013 (3) subdivided soft tissue liposarcoma into five categories, with atypical lipomatous tumour (ALT)/ well-differentiated liposarcoma (WDL) of the extremity being the most frequent subcategory. They are locally aggressive and have no potential for metastasis unless dedifferentiation occurs and are usually excised or, on occasion, observed. The term ALT is preferred to WDL when the lesion arises in the limbs or trunk, rather than retroperitoneum or mediastinum, but is generally considered synonymous as both have identical morphology, biological behaviour and genetics (3). Because their management is different from lipoma there is a requirement for confident distinction between these entities. However, even on histological analysis the diagnosis can be difficult and subjective (4) with multiple features (5-8) having been used to aid differentiation. In recent years, fluoresence in situ hybridisation (FISH) identification of murine double minute 2 (MDM2) gene amplification has become the gold standard for diagnosis of ALT/WDLs (9-11). There have been several studies assessing the ability of magnetic resonance imaging (MRI) to differentiate these lesions $(12,13)$. The previously well documented MRI accuracy for distinguishing these two tumours is in the region of $70 \%$ (12), with suggested reliable imaging discriminators being size and lipomatous content (13). It has however been noted that the imaging features of lipoma and ALT/WDL certainly overlap; with several studies concluding that discrimination using MRI is unreliable $(12,13)$. A further study suggested slightly improved sensitivity, specificity and accuracy in differentiating liposarcoma from lipoma with computer-assisted-diagnosis (CAD) (14). 
A potential CAD tool is radiomics-based textural analysis (TA), which is an imageprocessing technique that can assess tissue heterogeneity via variation in image signal intensity (both at and beyond that appreciated by the human eye). It quantifies the coarseness and regularity of the spatial distribution of pixel grey level values within normal and pathological tissue. Macroscopic heterogeneity in medical images may indirectly reflect underlying microscopic heterogeneity at the histopathological level. Recent studies have demonstrated clinical utility in tumour detection/grading, prognosis and treatment response $(15,16)$. Compared to computed tomography $(\mathrm{CT})$, MRI offers the advantages of improved soft tissue contrast resolution and a wealth of imaging data afforded by a multi-parametric approach. Recent studies have used magnetic resonance textural analysis (MRTA) for lesion detection, classification, treatment response evaluation and prognosis prediction in a number of different tumours such as breast, brain, and rectal cancers (17-19). There are very few studies of textural analysis reported in the musculoskeletal tumour literature (20-22). Several approaches to texture analysis have been described, with one widely published/validated approach being the filtration-histogram technique, where the filtration step extracts and enhances features at different scales followed by quantification of features using statistical and histogram analysis (23).

The purpose of this pilot study is to determine if heterogeneity of macroscopic fat can differentiate lipoma and ALT/WDL using MR radiomics-based texture analysis (MRTA). .

\section{Materials and methods:}

This was a retrospective review of 60 histologically confirmed ALT/WDL $=$ and lipoma. Cases were retrieved from the histopathology database of the Royal National Orthopaedic Hospital between December 2015 and May 2018. 30 consecutive cases of each diagnosis were selected retrospectively from the excision specimen data. All of the imaging was 
obtained at our own institution: recurrent tumours and those with inadequate or external MRI imaging were excluded. This study was approved by the Institute of Orthopaedics Research and Development department at the Royal National Orthopaedic Hospital, Stanmore, which determined there was no requirement for patient consent.

\section{Histology and MDM2 gene amplification analysis}

Retrospective review of reports by pathologists, experienced in the examination of soft tissue tumours, was performed, with final diagnosis based on both microscopic findings and FISH. For the former, a lipoma was defined as a lipomatous tumour composed of lobules of mature adipocytes separated by thin fibrous septa with no significant cytological atypia.ALT/WDL was diagnosed in a lipomatous tumour with thin fibrous septae containing scattered cells with dense hyperchromasia and / or cytological atypia . In the latter, a ratio greater than 2.0 on analysis of the FISH was considered to represent MDM2 amplification..

\section{Patient demographics}

Patient demographic data collected included age and gender.

\section{MR image analysis}

The routine MRI sequences included sagittal or coronal T1-weighted turbo spin echo (T1W TSE), sagittal or coronal short tau inversion recovery (STIR), coronal or sagittal T2-weighted fast spin echo (T2W FSE), axial proton density weighted fast spin echo (PDW FSE) and axial spectral adiabatic inversion recovery (SPAIR), with dedicated TR, TE and coil selection depending upon the body region being imaged. Post-contrast studies were not assessed. One musculoskeletal radiologist with 4 yearspost-fellowship experience in bone and soft tissue tumour MRI interpretation (IP), blinded to the final diagnosis, reviewed each of the 60 cases with documentation of the site of lesion, maximal lesion dimension, lesion depth relative to the fascia, as well as an estimation of the percentage of fat within the lesion. Depth relative to 
fascia was defined as either completely deep to deep fascia (sub-fascial) or superficial to/traversing deep fascia. A macroscopic approximation of the volume of fat within the lesion was undertaken by the reader and defined as having either "near complete intrinsic fat signal' or 'significant non-fat signal components/ septations'..

The authors considered that the lesions were "typical" examples of lipomas and ALT/WDLs, based on their daily clinical practice and available literature, such that a robust evaluation of MRTA in a representative population could be undertaken.MR-textural analysis (MRTA) The MRI reader selected both a single coronal T1 and single axial PDW FSE MRI image of the lesion that appeared to contain the most homogenous fat signal. These slices were saved as DICOM files and sent for MRTA analysis. The DICOM images were uploaded to commercially available research software (TexRAD, part of Feedback Medical Ltd Cambridge, UK - https://fbkmed.com/texrad-landing-2/). A region of interest (ROI) around the entire lesion was selected by a technician (RE), following appropriate education by the reader. The technician was also blinded to the diagnosis. Heterogeneity within all the MR images was evaluated using a filtration-histogram technique based textural analysis, where the filtration step comprised extracting and enhancing image features of different scales, followed by quantification of texture using statistical and histogram-based analysis. Specifically, image filtration used a band-pass Laplacian of Gaussian (LoG) filter (similar to non-orthogonal wavelet), which extracts and enhances features of different sizes and intensity-variation corresponding to the spatial scale of the filter (SSF in radius). The feature scales used were fine $(\mathrm{SSF}=2 \mathrm{~mm})$, medium $(\mathrm{SSF}=3,4$ and $5 \mathrm{~mm})$ and coarse $(\mathrm{SSF}=6 \mathrm{~mm})$. This was followed by quantification of texture using statistical and histogram-based parameters, reflecting different components of heterogeneity at each derived (feature-scale, SSF) image. Statistical and histogram-parameters included mean intensity (which reflects average brightness), standard-deviation (which reflects width of the histogram), entropy 
(which reflects irregularity), mean of positive pixels (which reflects average brightness of only positive pixel values), kurtosis (which reflects pointedness or peakedness) and skewness (which reflects asymmetry) (24). Figures 1 and 2 provide an illustration of the filtrationhistogram based MRTA for a lipoma and ALT/WDL.

\section{Statistical analysis}

The following analyses were applied to the entire study group and . two-sided tests with $p$ values less than 0.05 were considered to indicate a statistically significant difference:. patient demographics (age and sex), features of the lesion on imaging (size, location, depth versus fascia, and intra-lesional fat content) . Comparison was performedusing a non-parametric Mann Whitney test.

Comparison of lesion texture metrics for axial PD and coronal T1 was performed using nonparametric Mann Whitney test. Significant trends in texture metrics were visualised using box and whisker plots for the patient's groups and histograms for individual lesions.

$\underline{\text { Multivariate analysis: }}$

A composite score, combining (adding) the binary value of each significant univariate marker assessed the ability of the score to differentiate ALT/WDL from lipoma. Receiver operating characteristics (ROC) analysis established the diagnostic criteria (cut-off value), area under the ROC curve (AUC), sensitivity, specificity and p-value.

\section{Results}


Fifteen of $30(50 \%)$ of ALTs in this study had histopathological diagnosis confirmed with FISH MDM2 positivity, whilst 5 of $30(17 \%)$ of lipomas had confirmed FISH MDM2 negativity.8 (27\%) lipomas arose in the lower limb, 8 (27\%) in the upper limb and $14(47 \%)$ in the trunk and retroperitoneum, while 14 (47\%) ALTs arose in the lower limbs, 7 (23\%) in the upper limb and $9(30 \%)$ in the trunk and retroperitoneum (WDL).

No statistical difference was demonstrated with regards to age $(\mathrm{p}=0.442)$ or lesion size (0.111), the mean age for patients with ALT/WDLs was 61 years (median=60, range 27-over 89 years- according to institutional privacy rules all subjects $\geq 90$ years old, need to be grouped in a common age category) and with lipomas was 51 years (median $=51$, range 2976). The mean size of ALT/WDLs was $156.3 \mathrm{~mm}$ (median=148, range 41-417) and lipomas was $91.6 \mathrm{~mm}$ (median $=88$, range $36-161$ ). Ten of 30 ALT/WDLs measured $10 \mathrm{~cm}$ or less compared with 16 of 30 lipomas.

Table 1 summarizes the associations between the lipomatous lesions and the assessed binary imaging and non-imaging variables of interest in this study. Using histology and FISH as the gold standard, in addition to patient age and size of the lesion, statistically significant differences between lipomas and ALT/WDLs included location (site in body $(\mathrm{p}=0.021)$, depth from skin surface $(\mathrm{p}=0.001)$ and fat content $(\mathrm{p}=0.021)$.

Lesions containing near complete intrinsic fatty stroma (estimated as fatty content over 95\% of the volume of lesion using MRI), without solid masses or thick septation were observed in both patient groups (27 of 30 (90\%) lipomas (figure 1 and 3) compared to 17 of $30(56.7 \%)$ of ALT/WDL (figure 2).

In our patient population, 9 of $36(25 \%)$ texture parameters were found to have significant differences between the two patient populations on axial PD MRTA (Table 2). Significant differences were demonstrated over the range of textural parameters including for mean 
values at fine texture analysis (spatial scale filter $-\mathrm{SSF}=2, \mathrm{p}=0.015$ ). The most significant results were obtained at the medium and coarse texture scales, with p-values for mean and kurtosis on axial PD MRTA as follows: mean $\mathrm{p}=0.012$, kurtosis $\mathrm{p}=0.046$ at $\mathrm{SSF}=3$; mean $\mathrm{p}=0.008$, kurtosis $\mathrm{p}=0.023$ at $\mathrm{SSF}=4$; mean $\mathrm{p}=0.004$, kurtosis $\mathrm{p}=0.012$ at $\mathrm{SSF}=5$ and mean $\mathrm{p}=0.003$, kurtosis $\mathrm{p}=0.019$ at $\mathrm{SSF}=6$ respectively. Box and whisker plots illustrate the higher mean values for lipoma versus ALT/WDL on axial PD imaging (Figure 5).

This compares with 2 of $36(5.5 \%)$ of texture parameters with significant differences between the two patient populations on coronal T1 MRTA, which included kurtosis at medium texture scale $(\mathrm{SSF}=5, \mathrm{p}=0.041)$ and kurtosis at coarse texture scale $(\mathrm{SSF}=6, \mathrm{p}=0.046)$ (Table 3). Box and whisker plots illustrate the lower values for lipoma versus ALT/WDL with some negative values for kurtosis demonstrated in the lipoma group on T1-weighted imaging (Figure 6).

A coarse filtered $(\mathrm{SSF}=6)$ texture on axial PD MRI quantified as mean intensity (best univariate texture-marker) (see Table 2) with a cut-off value of <304 identified patients with ALT/WDL from lipoma with a sensitivity and specificity of $70 \%(\mathrm{AUC}=0.73, \mathrm{p}=0.003)$

A composite (risk) score combining the most significant univariate texture-marker, patient, and imaging parameters was undertaken. This included axial PD-MR texture parameter (mean intensity at coarse filtered texture scale $[\mathrm{SSF}=6]$ ), location (lower limb/retroperitoneal), depth (deep to fascia) and fat content (significant non-fat signal components/ septation) and identified patients with ALT from Lipoma with a AUC $=0.8$ $(\mathrm{p}<0.001)$. In particular, the presence of any 2 or more these four variables was associated with ALT with a sensitivity of $90 \%$ and specificity of $60 \%$. 


\section{Discussion}

In this study, we demonstrated statistically significant difference in the textural parameters of lipomas and ALTs when assessing the most 'macroscopically' fatty components of these lesions. Further research is needed to determine if MRTA can provide reliable, non-invasive distinction of these lesions.

Given that the histomorphological distinction of lipoma and ALT/WDL is relatively subjective, the consistent amplification of MDM2 detected by FISH has now become the gold standard, with reported $100 \%$ sensitivity and specificity (25). However, MR imaging features have documented only moderate accuracy for predicting the final diagnosis, compared to pathology. Ryan at al (12) suggested that the accuracy is in the region of $73 \%$ whilst Brisson et al (13) documented a sensitivity of $91 \%$, specificity of $37 \%$ and positive predictive value of 47\%. Prior to MDM2 FISH O'Donnell et al (26) suggested accuracy was in the region of $69 \%$ based on pathologic diagnosis using WHO criteria, whilst Gaskin et al (27) demonstrated an $83 \%$ specificity and a $38 \%$ PPV.

Therefore, there is a requirement to improve the differentiation of these entities noninvasively using MRI. In the present study, we evaluated intra-tumoural heterogeneity using MRI radiomics-based textual analysis to discriminate lipoma from ALT/WDL. To our knowledge, this has not been studied previously as a standalone tool. However, computer assisted diagnosis (CAD) utilising grey level co-occurrence, textural and morphological features has been assessed against radiologists' diagnosis of lipoma and liposarcoma, with CAD showing superior sensitivity, specificity and accuracy (14,). The authors (14) compared lipoma and high grade liposarcoma, which usually show different imaging appearances, making the performance of CAD in the current study, assessing lesions which are frequently 
indistinguishable on MRI, even more impressive. A more recent study suggests that using a combination of $\mathrm{T} 1$ and $\mathrm{T} 2 \mathrm{~W}$ images, the radiomic model showed even greater sensitivity (0.74) and specificity (0.88), and further outperformed radiologists (28). There are numerous published studies using textural analysis in other types of tumour however, the technique has been infrequently used in the musculoskeletal system. One study attempted to try to differentiate enchondroma from low-grade chondrosarcoma using 3-D-based MRI textural analysis. It found statistically significant differences in four of 20 textural parameters, including entropy and uniformity of positive pixel distribution of (UPP) on T1-weighted (T1W) imaging, and kurtosis and skewness on post contrast T1W imaging (22).

Given that our cases had all been referred to a tertiary centre for either consultation on the imaging features or due to the complexity of the surgery, all cases showed a degree of 'complexity'. However, the MRI imaging features and their statistical significance appear comparable those documented in the literature $(29,22$. Consistent with previous reports $(3$, 11, 13, 30-32), no gender predilection was identified between the patient groups; patients tended to be older (but with similar age ranges) and lesions generally larger in the group with ALT/WDLs. Using MRI, differentiation of lipoma and ALT/WDL remains poor, with previously documented accuracy below $80 \%$ likely reflecting overlapping imaging appearances. Our study suggests that assessment of several texture parameters may be a promising future approach: 9 of $36(25 \%)$ texture parameters were found to be significant discriminators of the two patient populations on axial PD imaging, but only 2 of $36(5.5 \%)$ on coronal T1-weighted images. The cause and significance of the discrepancy, which may be determined by the imaged plane or MRI sequence, is uncertain. It may in part represent the 'heterogeneous' nature of proton density imaging that comprises both T1 and T2weighting, supported by recent literature where addition of $\mathrm{T} 2 \mathrm{~W}$ together with $\mathrm{T} 1 \mathrm{~W}$ images further improved sensitivity and specificity (28). Fine, medium and coarse texture parameters 
had the potential to discriminate the two patient populations, particularly medium and coarse texture analysis quantified as mean intensity and kurtosis. It appears that higher mean and lower or negative kurtosis could potentially indicate a lipoma rather than ALT/WDL. The lower or negative kurtosis indicates that there is less visual contrast in the lesion, which intuitively corresponds with the gross radiological assessment of tumours. Higher mean values in lipoma suggest that they are more uniformly hyperintense and contain less contrast, compared to ALT/WDLs, which contain more intrinsic contrast: again, this would be anticipated given the intrinsic composition of these tumours. Just as the "macroscopic" imaging assessment of these lipomatous tumours relies on identification of non-fatty regions, so the computer assisted assessment of these textures relies on "microscopic" tumour heterogeneity. This is similar to the finding that several coarseness factors were able to discriminate enchondroma from low-grade chondrosarcoma (22). As in our study, kurtosis was also implicated as a statistically significant discriminator (22).

The best univariate texture marker was mean intensity at coarse textures. A cut off value of $<304$ has a sensitivity and specificity for differentiating ALT and lipoma of $70 \%$ which is comparable to the performance of radiologists $(13,26,27)$ and CAD $(14,28)$. Multivariate analysis of the most statistically significant parameters showed slight improvement, with an AUC of $0.8(\mathrm{p}<0.001)$, which is similar to the most recent radiomic study utilizing $\mathrm{T} 1$ and $\mathrm{T} 2$ weighted images (28).

There are some limitations in this study. The number of patients was rather small. However, equal numbers of each confirmed diagnosis, which helps reduce confidence intervals in statistical calculations, were used and . statistically significant outputs obtained. Given that the imaging was obtained on a single MRI machine, bias in the sample study should be reduced: bias may be introduced however, given the different locations of the tumours and 
the different coils and scanning parameters required to image them. The manual definition of the tumour boundaries in the semi-automated segmentation process is subjective and may introduce error.The specificity of the technique may be improved by drawing smaller ROIs in homogeneous areas, more accurately identifying microscopic heterogeneity. In this study, the ROI was drawn around the entire lesion on single slices. Although this was intentional, and assessedthe most macroscopically 'fatty' component, it excludes the most heterogenous components of the lesion. These could also be included in analysis of the whole tumour utilising volumetric data. . However, this may reduce specificity for the same reason as using a large ROI. We did not evaluate post contrast scans and do not feel they are helpful in the assessment of the well-differentiated lesions. Several authors have suggested that enhancement is predictive of $\operatorname{ALT}(33,34)$ but a recent study $(35)$ suggests similar accuracy of readers in lesion diagnosis with and without contrast studies. These authors went further to suggest that post contrast imaging did not significantly change confidence in diagnosis and in some cases led to changing of correct diagnoses prompting a conclusion that contrast enhancement in lipomatous lesions may be limited and occasionally misleading (35). Finally, a retrospective study design allows the evaluation of potential relationships between textual features and lesion characterisation, but can result in selection bias. There is potential for outof-data validation for these parameters which could provide further research results prior to validation of these findings in a prospective study for patients with fatty tumours..

MRI is clearly vital in the in the evaluation and pre-operative planning of lipomatous lesions, but there is the potential for improving pre-operative radiological diagnosis. Further assessment of MRI radiomics-based texture analysis of lipomatous and other tumours is warranted, given the preliminary positive outcome of this pilot study.

Texture heterogeneity quantified at fine, medium, coarse feature scales are significant differentiators of lipoma and ALT/WDL, in particular for medium and coarse texture scales 
for mean and kurtosis. Higher mean and lower or negative kurtosis can potentially distinguish lipoma from ALT/WDL. More significant differences were observed on axial PD compared to coronal T1-weighted images. Further research into formal heterogeneity assessment of lipomatous lesions in an attempt to improve non-invasive diagnostic accuracy is warranted. 


\section{References}

1. Myhre-Jensen O. A consecutive 7-year series of 1331 benign soft tissue tumors. Clinicopathologic data. Comparison with sarcomas. Acta Orthop Scand 1981; 52:287-293.

2. Dei Tos AP. Liposarcoma: new entities and evolving concepts. Ann Diagn Pathol $2000 ; 4: 252-266$.

3. International Agency for Research on Cancer, World Health Organisation, International Academy of Pathology, Bridge JA, Hogendoorn PC, Fletcher C. WHO Classifiation of Tumours of Soft Tissue and Bone 2013. 4th ed. IARC; 2013.

4. Coindre JM, Pedeutour F, Aurias A. Well differentiated and dedifferentiated liposarcomas. Virchows Arch 2010; 456:167-79.

5. Shimada S, Ishizawa T, Ishizawa K, Matsumura T, Hasegawa T, Hirose T. The value of MDM2 and CDK4 amplification levels using real-time polymerase chain reaction for the differential diagnosis of liposarcomas and their histologic mimickers. Hum Pathol 2006; 37:1123-1129.

6. Hostein I, Pelmus M, Aurias A, Pedeutour F, Mathoulin-Pelissier S, Coindre JM. Evaluation of MDM2 and CDK4 amplification by real-time PCR on paraffin waxembedded material: a potential tool for the diagnosis of atypical lipomatous tumours/well differentiated liposarcomas. J Pathol 2004; 202:95-102.

7. Sirvent N, Coindre JM, Maire G, Hostein I, Keslair F, Guillou L et al. Detection of MDM2-CDK4 amplification by fluorescence in situ hybridization in 200 paraffinembedded tumor samples: utility in diagnosing adipocytic lesions and comparison 
with immunohistochemistry and real-time PCR. Am J Surg Pathol 2007; 31:14761489.

8. Binh MB, Sastre-Garau X, Guillou L, de Pinieux G, Terrier P, Lagace R et al. MDM2 and CDK4 immunostainings are useful adjuncts in diagnosing welldifferentiated and dedifferentiated liposarcoma subtypes: a comparative analysis of 559 soft tissue neoplasms with genetic data. Am J Surg Pathol 2005; 29:1340-1347.

9. Kashima T, Halai D, Ye H, Hing SN, Delany D, Pollock R et al. Sensitivity of MDM2 amplification and unexpected multiple faint alphoid 12 (alpha 12 satellite sequences) signals in atypical lipomatous tumour. Mod Pathol 2012; 25:1384-96.

10. Weaver J, Downs-Kelly E, Goldblum JR, Turner S, Kulkarni S, Tubbs RR et al. Fluoresence in situ hybridization for MDM2 gene amplification as a diagnostic tool in lipomatous neoplasms. Mod Pathol 2008; 21:943-949.

11. Dei Tos AP, Doglioni C, Piccinin S, Sciot R, Furlanetto A, Boiocchi M et al. Coordinated expression and amplification of the MDM2, CDK4, and HMGI-C genes in atypical lipomatous tumours. J Pathol 2000; 190:531-536.

12. Ryan S, Visgauss J, Kerr D, Helmkamp J, Said N, Vinson E et al. The value of MRI in distinguishing subtypes of lipomatous extremity tumors needs reassessment in the era of MDM2 and CDK4 testing. Sarcoma 2018, article ID 1901896, 7 pages.

13. Brisson M, Kashima T, Delaney D, Tirabosco R, Clarke A, Cro S et al. MRI characteristics of lipoma and atypical lipomatous tumor/well-differentiated liposarcoma: retrospective comparison with histology and MDM2 gene amplification. Skeletal Radiol 2013; 42:635-647. 
14. Thornhill RE, Golfam M, Sheikh A, Cron GO, White EA, Werier J et al.

Differentiation of lipoma from liposarcoma on MRI using texture and shape analysis. Acad Radiol 2014; 21:1185-1194.

15. Davnall F, Yip CS, Ljungqvist G, Selmi M, Ng F, Sanghera B et al (2012) Assessment of tumor heterogeneity: an emerging imaging tool for clinical practice? Insights Imaging 3:573-589

16. Ganeshan B, Miles KA (2013) Quantifying tumour heterogeneity with CT. Cancer Imaging 13:140-149

17. Eliat PA, Olivie D, Saikali S, Carsin B, Saint-Jalmes H, de Certaines JD (2012) Can dynamic contrast-enhanced magnetic resonance imaging combined with texture analysis differentiate malignant glioneuronal tumors from other glioblastoma? Neurol Res Int 2012, 195176

18. Parikh J, Selmi M, Charles-Edwards G, Glendenning J, Ganeshan B, Verma H et al (2014) Changes in primary breast cancer heterogeneity may augment midtreatment MR imaging assessment of response to neoadjuvant chemotherapy. Radiology $272: 100-112$

19. De Cecco CN, Ganeshan B, Ciolina M, Rengo M, Meinel FG, Musio D et al (2015) Texture analysis as imaging biomarker of tumoral response to neoadjuvant chemoradiotherapy in rectal cancer patients studied with 3-T magnetic resonance. Invest Radiol 50:239-245

20. Mayerhoefer ME, Breitenseher MJ, Kramer J et al. Texture analysis for tissue discrimination on T1-weighted MR images of the knee joint in a multicentre study: transferability of texture features and comparison of feature selection methods and classifiers. J Magn Reason Imaging 2005; 22:674-680. 
21. Juntu J, Sijbers J, Van Dyck D et al. Bias field correction for MRI images. Computer recognition systems. Advances in soft computing 2005; 30:543-551.

22. Lisson CS, Lisson CG, Flosdorf K, Mayer-Steinacker R, Schultheiss M, von Baer A et al. Diagnostic value of MRI-based 3D texture analysis for tissue characterisation and discrimination of low-grade chondrosarcoma from enchondroma: a pilot study. Eur Radiol 2018; 28:468-477.

23. Miles KA, Ganeshan B, Hayball MP (2013) CT texture analysis using the filtrationhistogram method: what do the measurements mean? Cancer Imaging 13:400-406.

24. Miles KA. Quantifying tumour heterogeneity with CT. Cancer Imaging 2013; $13: 140-9$

25. Weaver J, Rao J, Goldblum et al. Can MDM2 analytical tests performed on core needle biopsy be relied upon to diagnose well-differentiated liposarcoma? Modern Pathology 2010; 23:1301-1306.

26. O’Donnell PW, Griffin AM, Edward WC et al. Can experienced observers differentiate between lipoma and well-differentiated liposarcoma using only MRI? Sarcoma 2013, article ID 982784, 6 pages.

27. Gaskin GM, Helms CA. Lipomas, lipoma variants, and well-differentiated liposarcomas (atypical lipomas): results of MRI evaluations of 126 consecutive fatty masses. American Journal of Roentgenology 2004; 182:733-739.

28. Vos M, Starmans PA, Timbergen MJM, van der Voort SR, Padmos GA, Kessels W et al. Radiomics approach to distinguish between well differentiated liposarcomas and lipomas on MRI. Br J Surg 2019; 106(13):1800-1809. 
29. Kransdorf MJ, Bancroft LW, Peterson JJ, Murphey MD, Foster WC, Temple HT. Imaging of fatty tumors: distinction of lipoma and well-differentiated liposarcoma. Radiology 2002; 224:99-104.

30. Evans HL. Atypical lipomatous tumor, its variants, and its combined forms: a study of 61 cases, with a minimum follow-up of 10 years. Am J Surg Pathol 2007; 31:1-14.

31. Murphey MD, Carroll JF, Flemming DJ, Pope TL, Gannon FH, Kransdorf MJ. From the archives of the AFIP: benign musculoskeletal lipomatous lesions. Radiographics $2004 ; 24: 1433-66$.

32. Donato M, Vanel D, Alberghini M, Mercuri M. Muscle fibers inside a fat tumor: a non-specific imaging finding of benignancy. Eur J Radiol 200; 72:27-9.

33. Knebel C, Neumann J, Schwaiger BJ, Karampinos DC, Pfeiffer D, Specht K et al. Differentiating atypical lipomatous tumors from lipomas with magnetic resonance imaging: a comparison with MDM2 gene amplification status. BMC Cancer 2019; 19(1):309.

34. Oghuri T, Aoki T, Hisaoka M et al. Differential diagnosis of benign peripheral lipoma from well-differentiated liposarcoma on MR imaging: is comparison of margins and internal characteristics useful? Am J Roentgenol 2003; 180: 1689-94.

35. Nardo L, Abdelhafez YG, Acquafredda F, Schiro S, Wong AL, Sarohia D et al. Qualitative evaluation of MRI features of lipoma and atypical lipomatous tumor: results from a multicenter study. Skeletal Radiol 2020 epub ahead of print. 


\section{Table legends}

Table 1 Summary of results for each patient and imaging variables in group with ALT/WDL and lipoma. Statistically significant results $(\mathrm{p}<0.05)$ highlighted in yellow. 
ALT/WDL(n) Lipoma(n) $\quad p$ value

\begin{tabular}{|c|c|c|c|c|}
\hline \multirow[t]{2}{*}{ Gender } & Male & 16 & 13 & 0.442 \\
\hline & Female & 14 & 17 & \\
\hline \multirow[t]{2}{*}{ Age } & $>60$ & 14 & 8 & 0.111 \\
\hline & $\leq 60$ & 16 & 22 & \\
\hline \multirow[t]{2}{*}{ Lesion size } & $>10 \mathrm{~cm}$ & 20 & 14 & 0.121 \\
\hline & $\leq 10 \mathrm{~cm}$ & 10 & 16 & \\
\hline \multirow[t]{2}{*}{ Location } & UL/ trunk & 11 & 20 & 0.021 \\
\hline & $\mathrm{LL} /$ retroperitoneum & 19 & 10 & \\
\hline \multirow[t]{2}{*}{ Depth } & Deep to fascia & 27 & 13 & 0.001 \\
\hline & Superficial/ traverses & 3 & 15 & \\
\hline Fat & Near complete & & & \\
\hline \multirow[t]{4}{*}{ content } & intrinsic fat signal & 18 & 26 & 0.021 \\
\hline & Significant non-fat & & & \\
\hline & signal components/ & & & \\
\hline & septation & 12 & 4 & \\
\hline
\end{tabular}


Table 2 Summary of results (median) for axial PD MRTA heterogeneity variables in group with ALT/WDL and lipoma. Statistically significant results $(\mathrm{p}<0.05)$ highlighted in yellow.

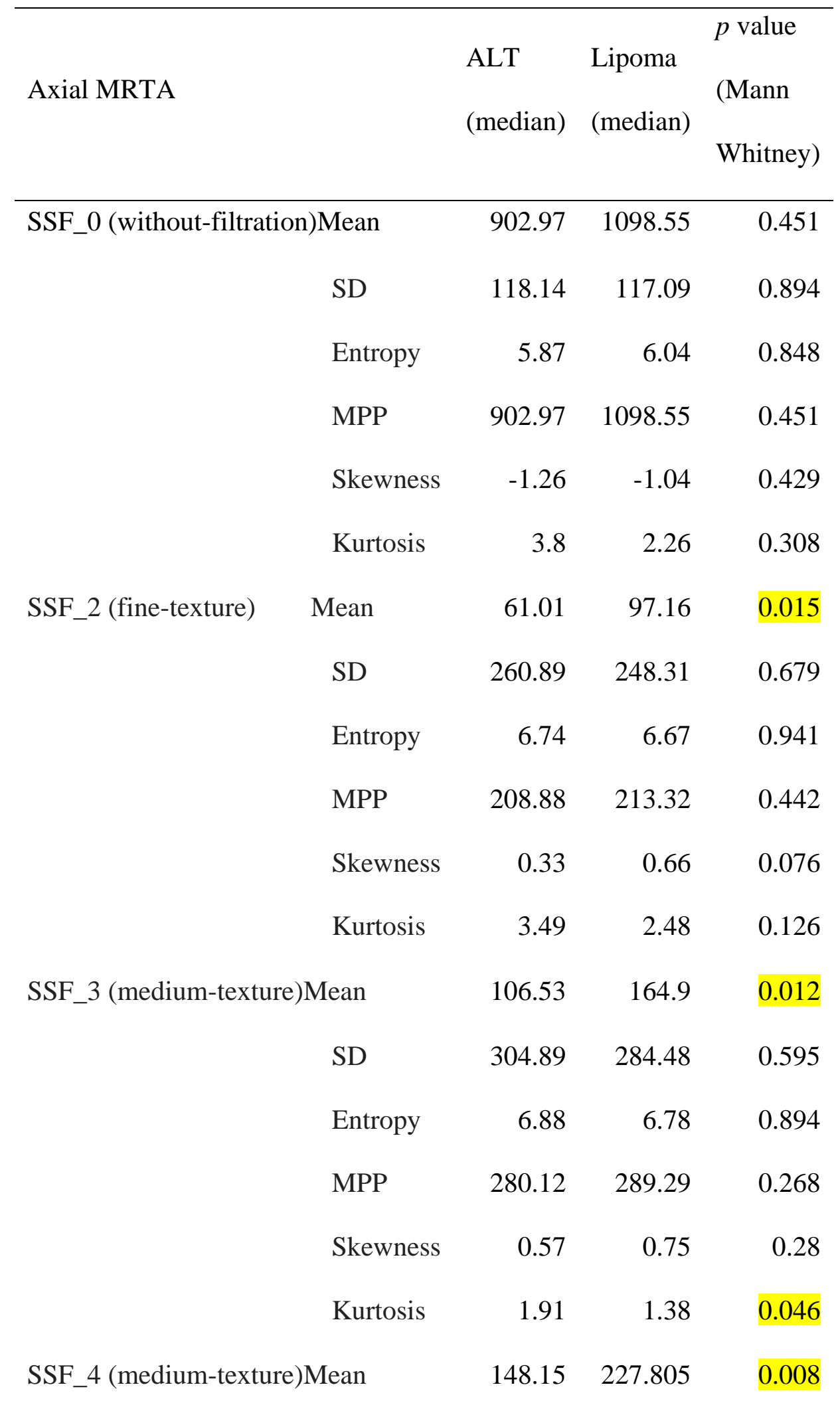




\begin{tabular}{|c|c|c|c|c|}
\hline & SD & 337.5 & 335.49 & 0.469 \\
\hline & Entropy & 7.02 & 6.87 & 0.813 \\
\hline & MPP & 331.68 & 389.65 & 0.147 \\
\hline & Skewness & 0.47 & 0.6 & 0.6 \\
\hline & Kurtosis & 1.4 & 0.61 & 0.023 \\
\hline SSF_5 (medium-texture) & Mean & 201.15 & 302.45 & 0.004 \\
\hline & SD & 353.09 & 365.86 & 0.391 \\
\hline & Entropy & 7.03 & 6.94 & 0.734 \\
\hline & MPP & 348.95 & 473.45 & 0.074 \\
\hline & Skewness & 0.39 & 0.48 & 0.9 \\
\hline & Kurtosis & 0.99 & 0.42 & 0.012 \\
\hline SSF_6 (coarse-texture) & Mean & 256.6 & 385.1 & 0.003 \\
\hline & SD & 363.94 & 382.04 & 0.337 \\
\hline & Entropy & 7.02 & 7.1 & 0.61 \\
\hline & MPP & 368.26 & 538.37 & 0.053 \\
\hline & Skewness & 0.38 & 0.37 & 0.584 \\
\hline & Kurtosis & 0.35 & 0.04 & 0.019 \\
\hline
\end{tabular}

Table 3 Summary of results (median) for coronal T1 MRTA heterogeneity variables in group with ALT/WDL and lipoma. Statistically significant results $(\mathrm{p}<0.05)$ highlighted in yellow.

\begin{tabular}{lccc}
\hline & ALT/WDL & Lipoma & $p$ value \\
Coronal MRTA & (median) & (median) & (Mann \\
& & & Whitney) \\
& & & \\
\hline SSF_0 (without-filtration)Mean & 1154.98 & 1172.3 & 0.657
\end{tabular}




\begin{tabular}{|c|c|c|c|c|}
\hline & SD & 152.47 & 107.39 & 0.16 \\
\hline & Entropy & 6.04 & 5.71 & 0.061 \\
\hline & MPP & 1154.98 & 1172.3 & 0.657 \\
\hline & Skewness & -1.13 & -1.19 & 0.171 \\
\hline & Kurtosis & 2.61 & 2.25 & 0.311 \\
\hline SSF_2 (fine-texture) & Mean & 44.25 & 58.04 & 0.469 \\
\hline & SD & 289.47 & 210.1 & 0.209 \\
\hline & Entropy & 6.8 & 6.45 & 0.141 \\
\hline & MPP & 227.63 & 161.95 & 0.46 \\
\hline & Skewness & -0.08 & 0.39 & 0.51 \\
\hline & Kurtosis & 4.91 & 4.83 & 0.469 \\
\hline SSF_3 (medium-texture) & Mean & 79.36 & 105.35 & 0.408 \\
\hline & SD & 331.36 & 252.89 & 0.261 \\
\hline & Entropy & 7 & 6.64 & 0.174 \\
\hline & MPP & 271.74 & 239.91 & 0.554 \\
\hline & Skewness & 0.23 & 0.59 & 0.308 \\
\hline & Kurtosis & 3.48 & 2.38 & 0.162 \\
\hline SSF_4 (medium-texture) & Mean & 105.27 & 151.73 & 0.391 \\
\hline & SD & 388.42 & 300.99 & 0.322 \\
\hline & Entropy & 7.25 & 6.78 & 0.181 \\
\hline & MPP & 332.43 & 282.39 & 0.657 \\
\hline & Skewness & 0.27 & 0.44 & 0.237 \\
\hline & Kurtosis & 2.35 & 1.35 & 0.061 \\
\hline SSF_5 (medium-texture) & Mean & 129.72 & 205.43 & 0.329 \\
\hline & SD & 406.82 & 358.63 & 0.315 \\
\hline
\end{tabular}




$\begin{array}{lrrrr} & \text { Entropy } & 7.35 & 6.89 & 0.206 \\ & \text { MPP } & 345.44 & 330.42 & 0.767 \\ & \text { Skewness } & 0.27 & 0.41 & 0.359 \\ & \text { Kurtosis } & 1.69 & 0.64 & 0.041 \\ \text { SSF_6 (coarse-texture) } & \text { Mean } & 151.91 & 264.06 & 0.28 \\ & \text { SD } & 434.65 & 400.26 & 0.315 \\ & \text { Entropy } & 7.33 & 7.02 & 0.188 \\ & \text { MPP } & 390.61 & 402.14 & 0.918 \\ & \text { Skewness } & 0.23 & 0.34 & 0.589 \\ & \text { Kurtosis } & 1.03 & 0.46 & 0.046\end{array}$




\section{Figure Legends}

Fig. 1 Coronal T1 (left) and axial PD (right) MRI images with ROI included (thin blue line) and corresponding filtered texture maps fine (top right), medium (bottom left) and coarse (bottom right) texture heterogeneity of a molecular pathology proven lipoma.
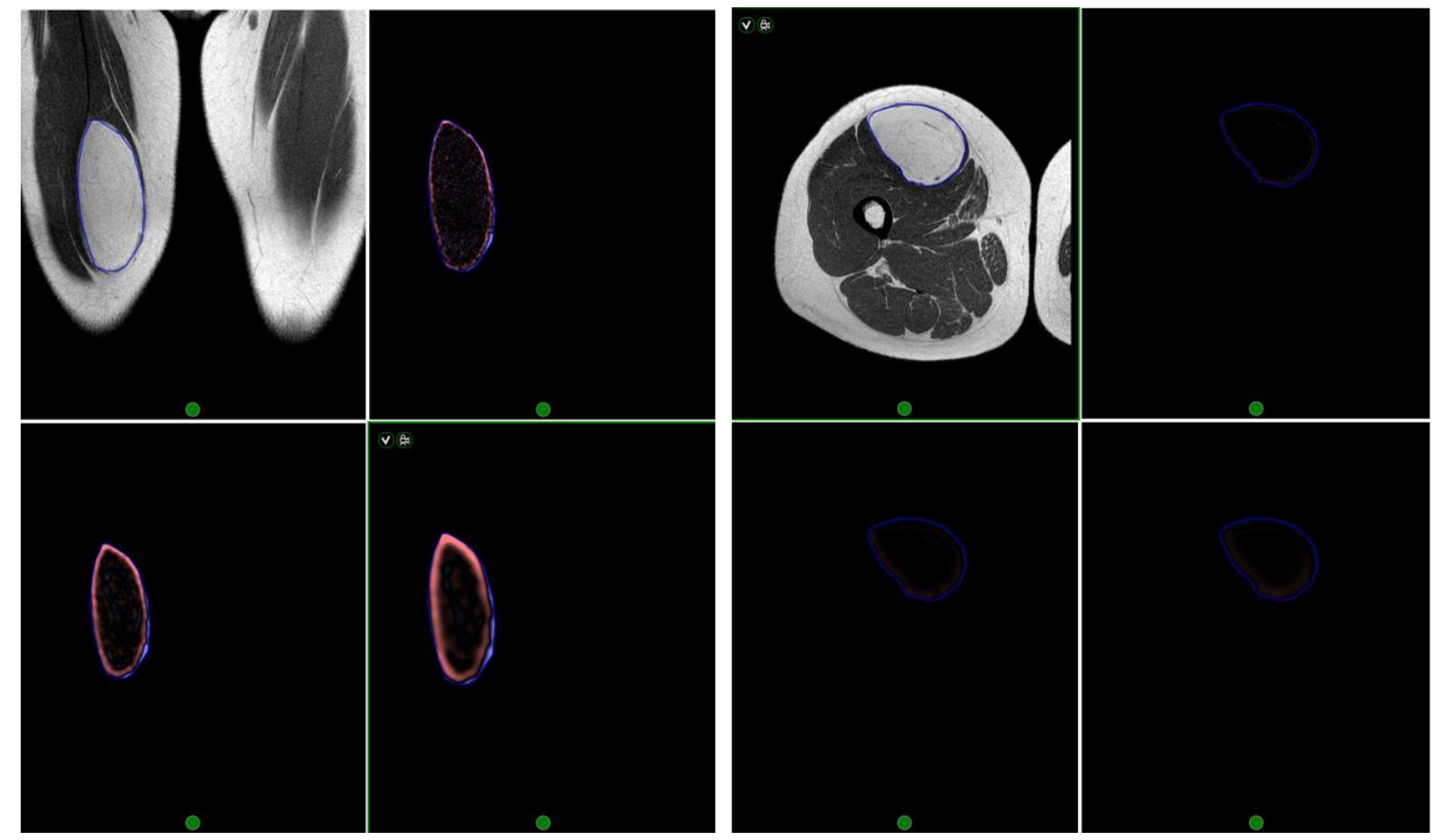

Fig. 2 Coronal T1 (left) and axial PD (right) MRI images with ROI included (thin blue line) and corresponding filtered texture maps fine (top right), medium (bottom left) and coarse (bottom right) texture heterogeneity of a molecular pathology proven ALT. expressing MDM2. 

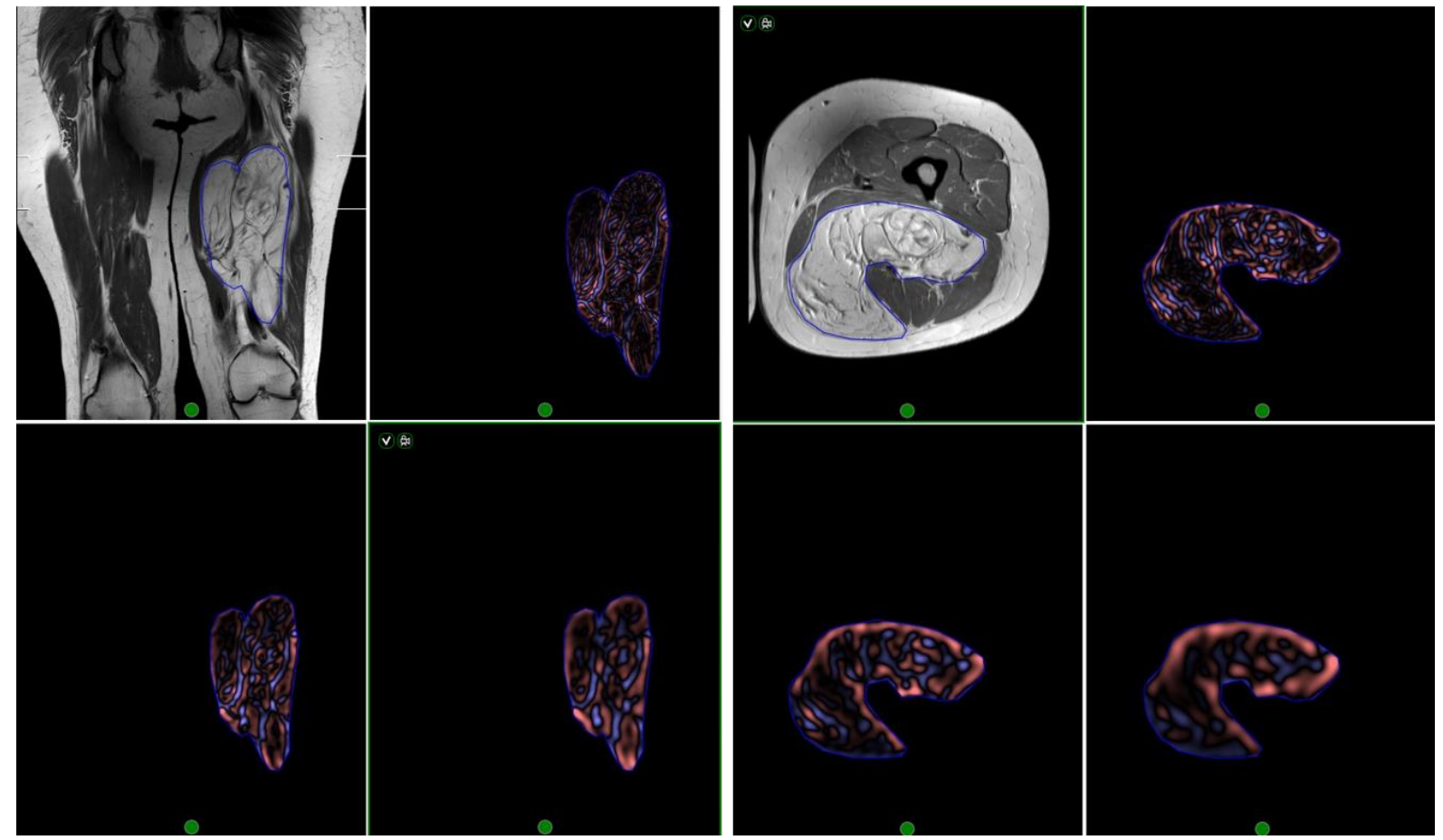

Fig. 3 Coronal T1 (above - left) and axial PD (below - left) MRI images and corresponding radiomics-based texture heterogeneity analysis - filtered map at medium scale (middle) and quantification using histogram-analysis (right) for a molecular pathology proven lipoma.
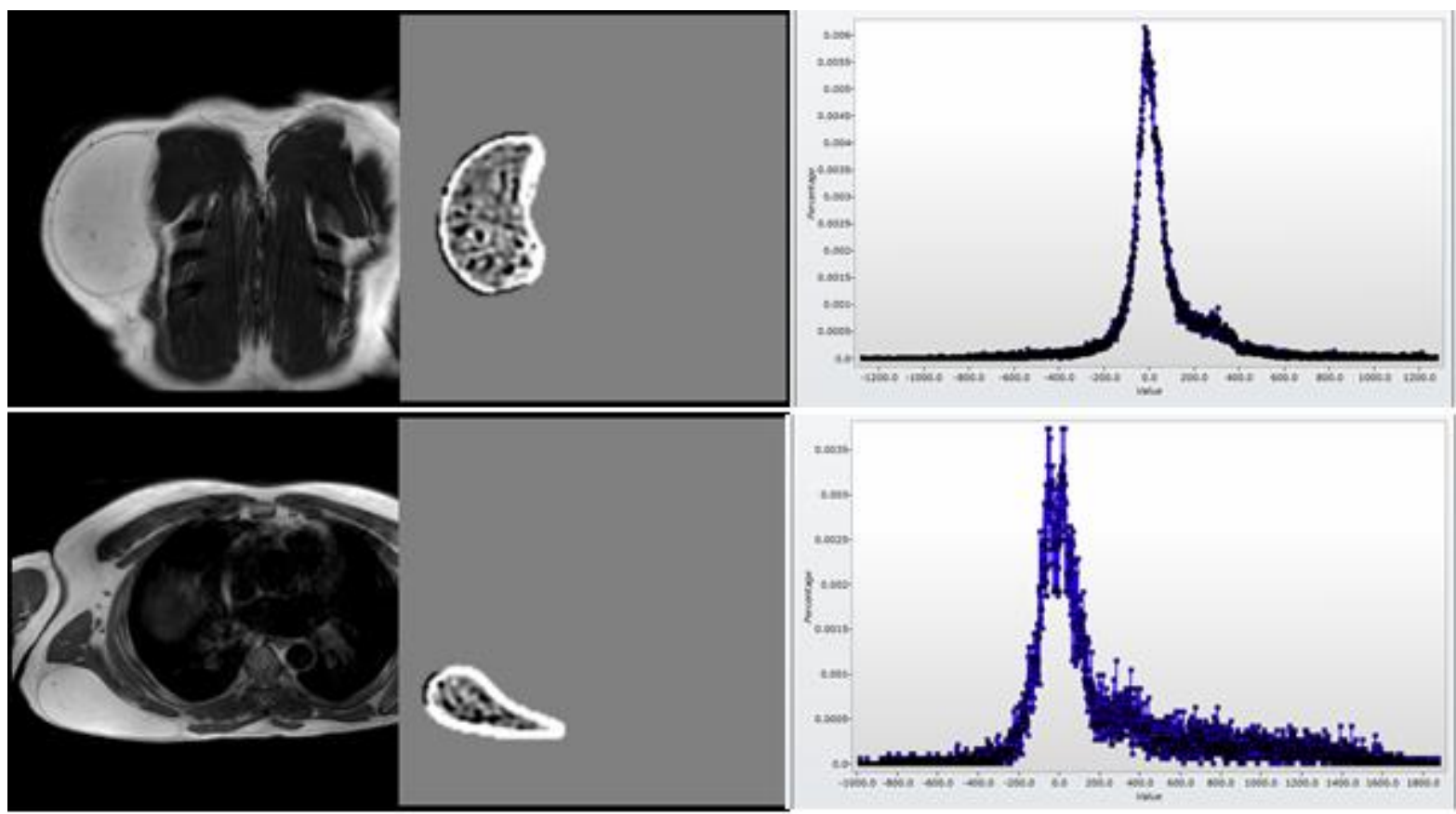
Fig. 4 Coronal T1 (above - left) and axial PD (below - left) MRI images and corresponding radiomics-based texture heterogeneity analysis - filtered map at medium scale (middle) and quantification using histogram-analysis (right) for a molecular pathology proven ALT.
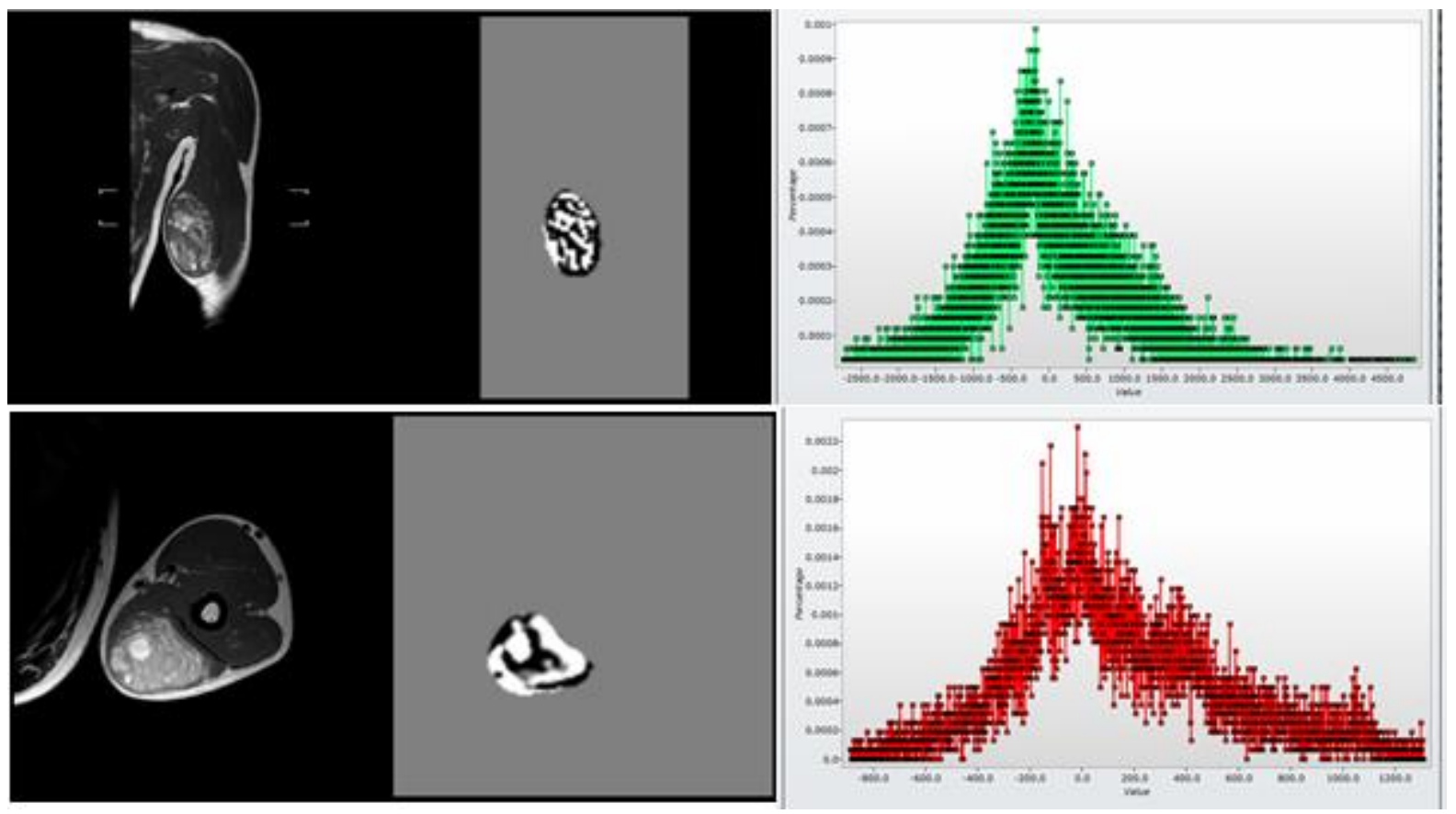

Fig. 5 Box and whisker plot demonstrating axial PD mean values for ALT/WDL (0) and lipoma (1). Note the higher mean values in lipoma. 


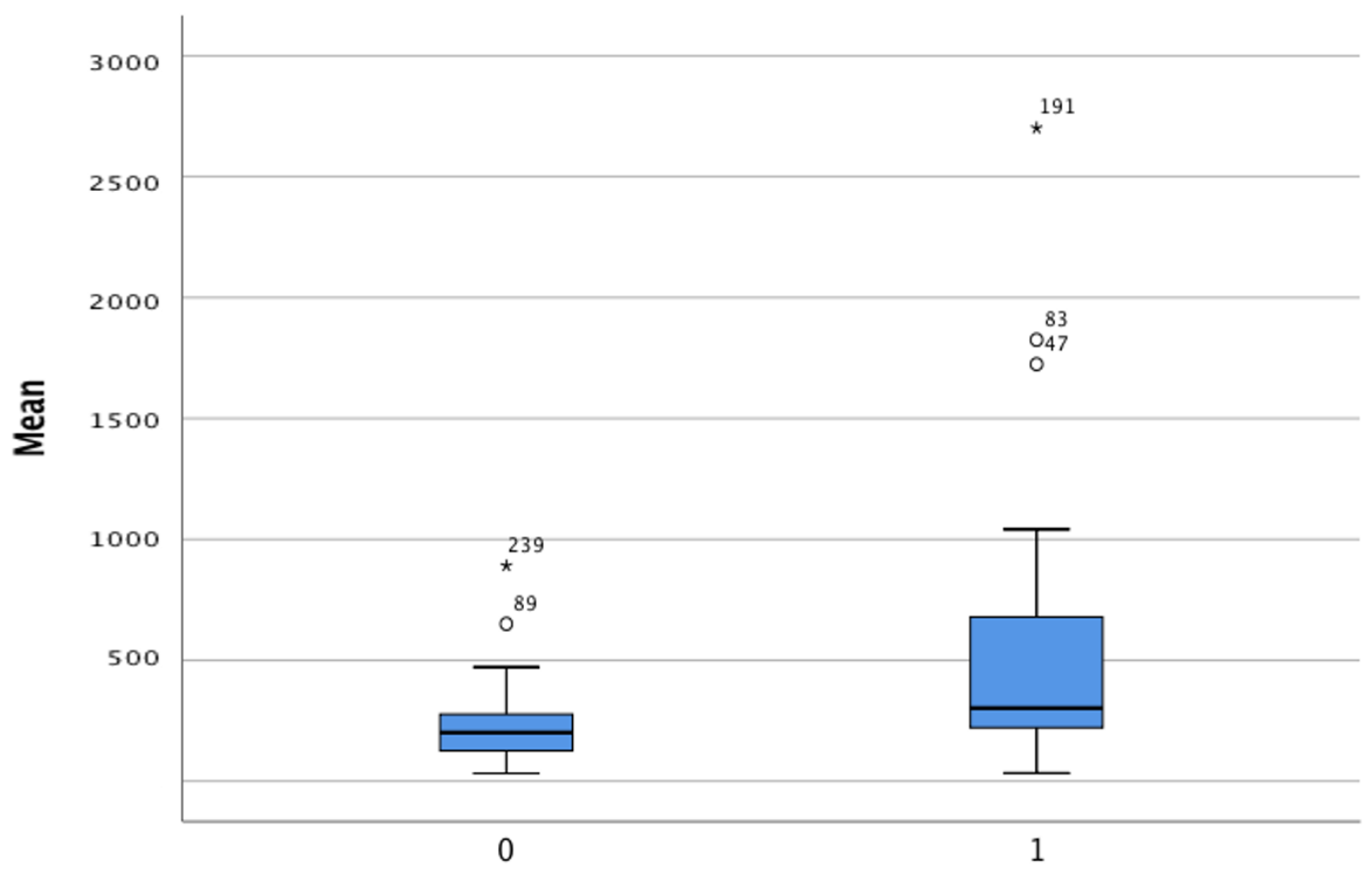

Fig. 6 Box and whisker plot demonstrating coronal T1 kurtosis values for ALT/WDL (0) and lipoma (1). Note the lower, with some negative values, for kurtosis for lipoma. 


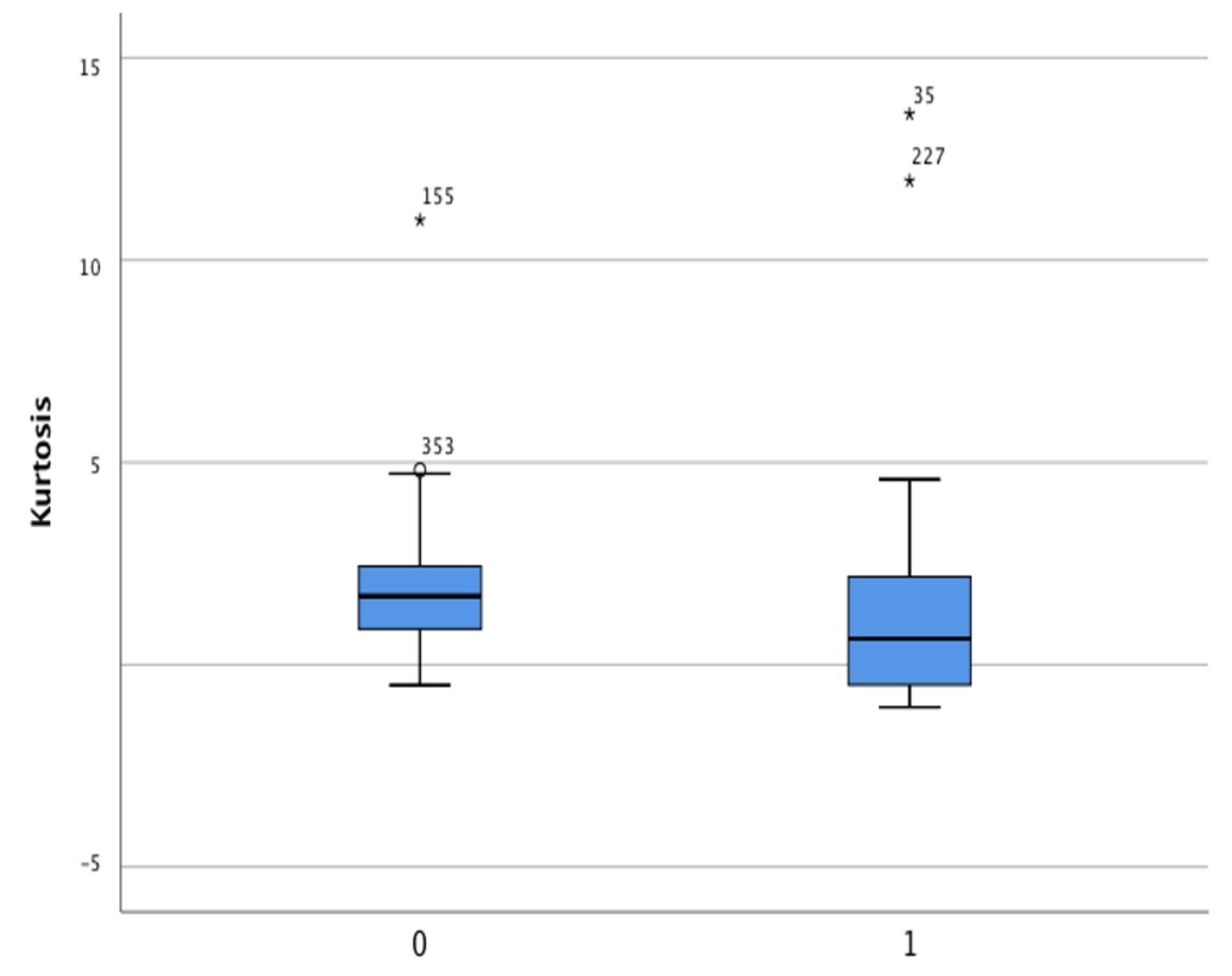

